\title{
On Strong Closure of Sets of Feasible States Associated with Families of Elliptic Operators
}

\author{
O. Zaytsev
}

\begin{abstract}
The closure of sets of feasible states for systems of elliptic equations in the strong topology of the Cartesian product $\left[H_{0}^{1}(\Omega)\right]^{m}$ of Sobolev spaces is considered. For $m=2$ and $\Omega \subset \mathbb{R}^{2}$, it is shown that there is a family of linear elliptic operators of the type $\operatorname{div}\left(\chi \mathcal{A}^{1}+\right.$ $\left.(1-\chi) \mathcal{A}^{2}\right) \nabla$, where $\chi$ belongs to the set of all characteristic functions of measurable subsets of $\Omega$, such that there does not exist a larger family of operators of the type $\operatorname{div} \mathcal{A} \nabla$ for which the sets of feasible states coincide with the closure of the original ones.
\end{abstract}

Keywords: Strong closure, feasible states, elliptic operators, systems of elliptic equations AMS subject classification: Primary $49 \mathrm{~J} 45$, secondary $35 \mathrm{~J} 50,49 \mathrm{~J} 20$

\section{Introduction}

In [6] there is studied an optimal control problem of the form

$$
I(u):=\int_{\Omega}(\operatorname{grad}(u-v))^{2} d x \rightarrow \min
$$

where $v \in H_{0}^{1}(\Omega)$ is fixed, $u$ is a solution of a diffusion equation in a bounded open subset $\Omega$ of $\mathbb{R}^{n}$

$$
-\operatorname{div}(a \operatorname{grad} u)=f \quad \text { in } \Omega, u \in H_{0}^{1}(\Omega)
$$

with $f \in H^{-1}(\Omega)$ and $a \in L^{\infty}(\Omega)$ satisfying

$$
\left.\begin{array}{l}
0<\lambda_{1} \leq a(x) \leq \lambda_{2} \quad \text { for a.e. } x \in \Omega \\
a(x) \in\left\{\mu_{1}, \ldots, \mu_{r}\right\} \quad \text { for a.e. } x \in \Omega \\
\text { meas }\left\{x \in \Omega \mid a(x)=\mu_{q}\right\} \leq k_{q}, \sum_{q=1}^{r} k_{q} \geq \operatorname{meas} \Omega
\end{array}\right\}
$$

where $\lambda_{1}, \lambda_{2}, \mu_{1}, \ldots, \mu_{r}, k_{1}, \ldots, k_{r}$ are fixed constants. As noted in [6], the problems involving grad $u$ non-linearly are more realistic than those which contain only $u$. We refer to [1] for other problems involving grad $u$ non-linearly.

O. Zaytsev: University of Latvia, Institute of Mathematics and Computer Science, 29 Rainis Boulevard, LV-1459, Riga, Latvia 
Let $S$ denote the set of all functions $a$ satisfying (2). In [6] the equality

$$
\begin{aligned}
\operatorname{clos}_{s} & \left\{u \in H_{0}^{1}(\Omega) \mid-\operatorname{div}(a \operatorname{grad} u)=f \text { in } \Omega, a \in S\right\} \\
& =\left\{u \in H_{0}^{1}(\Omega) \mid-\operatorname{div}(b \operatorname{grad} u)=f \text { in } \Omega, b \in \overline{\operatorname{co}} S\right\} \quad \text { for all } f \in H^{-1}(\Omega)
\end{aligned}
$$

is proved where $\mathrm{cl}$, denotes the operation of the strong closure in $H_{0}^{1}(\Omega)$ and $\overline{c o}$ stands for the symbol of the closed convex hull in $L^{2}(\Omega)$. Here we would like to refer to [4] for more general results (see also $[3,5]$ ).

Equality (3) gives a good basis for further analysis. For instance, one can pass to the extended problem with $\overline{c o} S$ instead of $S$ and, by using the convexity of $\overline{c o} S$, one can derive necessary conditions of optimality as it was done in [6]. In addition, the convex set $\overline{c o} S$ is more preferable from the point of view of further numerical evaluation.

It is very important to know whether an equality of type (3) holds for other families and, especially, for families of linear systems of elliptic operators in which the number $m$ of unknown functions is equal to or greater than the number $n$ of independent variables. More precisely, for $m \geq n \geq 2$, let $\mathfrak{A}$ be the family of all elliptic operators $A$ of the type

$$
A=\operatorname{div} \mathcal{A} \nabla
$$

where $\mathcal{A}$ is a positive definite $(m n \times m n)$-matrix with entries from $L^{\infty}(\Omega)$ taking its values in a finite set $\left\{\mathcal{A}^{1}, \ldots, \mathcal{A}^{r}\right\}$ of positive definite $(m n \times m n)$-matrices $\mathcal{A}^{1}, \ldots, \mathcal{A}^{r}$.

For $\tilde{f} \in\left[H^{-1}(\Omega)\right]^{m}$ and $\tilde{u} \in\left[H_{0}^{1}(\Omega)\right]^{m}$, define

$$
Z(\mathfrak{A}, \tilde{f})=\left\{u \in\left[H_{0}^{1}(\Omega)\right]^{m} \mid A u=\tilde{f}, A \in \mathfrak{A}\right\} \quad \text { (set of feasible states) }
$$

and

$$
F(\mathfrak{A}, \tilde{u})=\left\{A \tilde{u} \in\left[H^{-1}(\Omega)\right]^{m} \mid A \in \dot{\mathfrak{A}}\right\} .
$$

Now the question, we are interested in, can be formulated as follows: Does there exist a family $\mathfrak{B}$ of operators of the type (4) such that

$$
\operatorname{clos}_{s} Z(\mathfrak{A}, f)=Z(\mathfrak{B}, f) \quad \text { for all } f \in\left[H^{-1}(\Omega)\right]^{m} \text { ? }
$$

Since the operators from $2 A$ are uniformly continuous and coercive, the previous question is equivalent to the following: Does there exist a family $\mathfrak{B}$ of operators of the type (4) such that

$$
\operatorname{clos}_{s} F(\mathfrak{A}, u)=F(\mathfrak{B}, u) \quad \text { for all } u \in\left[H_{0}^{1}(\Omega)\right]^{m} ?
$$

As it turns out, the answer to this question is, in general, negative. We prove this by constructing a corresponding example of the family $\mathfrak{A}$ for the case when $r=m=n=2$. This example shows that for optimal control problems with non-weakly continuous cost functionals (for instance, with functionals of the type (1)) governed by elliptic systems of the type (4) possible extensions depend, in general, not only on the family 2 but on the functional $I$ and the right-hand side $f$, too, even if the cost functional and the right-hand side do not depend on controls. 


\section{Preliminaries and statement of the result}

Let $\mathbb{R}^{n}$ denote the $n$-dimensional Euclidean space. Let $\Omega$ be a bounded domain in $\mathbb{R}^{n}$ with Lipschitz boundary. The symbol $\Omega^{\prime} \subset \subset \Omega$ means that $\Omega^{\prime}$ is a subdomain of $\Omega$ such that $\operatorname{dist}\left(\Omega^{\prime}, \partial \Omega\right)>0$. We will denote by $C_{0}^{\infty}(\Omega)$ the set of functions with compact support in $\Omega$ having all derivatives of arbitrary order continuous in $\Omega$. The notations $L^{p}(\Omega) \quad(p=2, \infty)$ mean the usual Lebesgue spaces. $H^{1}(\Omega)$ is the Sobolev space of functions $f \in L^{2}(\Omega)$ such that $\frac{\partial f}{\partial x_{i}} \in L^{2}(\Omega)(1 \leq i \leq n)$ with the usual norm. $H_{0}^{1}(\Omega)$ is the closure of $C_{0}^{\infty}(\Omega)$ in $H^{1}(\Omega) . H^{-1}(\Omega)$ is the dual space of $H_{0}^{1}(\Omega)$. We use the notation $\mathcal{D}^{\prime}(\Omega)$ to denote the space of distributions on $\Omega$.

For the sake of brevity, we put $V=\left[H_{0}^{1}(\Omega)\right]^{m}$ and $V^{*}=\left[H^{-1}(\Omega)\right]^{m}$. Let $\mathcal{A}=$ $\left\{a_{i j k l}\right\}_{\substack{1 \leq i, k \leq m \\ 1 \leq j, 1 \leq n}}$ be an $(m n \times m n)$-matrix satisfying the conditions

$$
\begin{aligned}
& \nu_{1} \xi^{2} \leq(\mathcal{A}(x) \xi, \xi) \leq \nu_{2} \xi^{2} \quad \text { for a.e. } x \in \Omega \text { and all } \xi \in \mathbb{R}^{m \times n} \\
& \mathcal{A} \in\left[L^{\infty}(\Omega)\right]^{m \times m n}
\end{aligned}
$$

where $\nu_{1}, \nu_{2}>0$. We relate to $\mathcal{A}$ the operator $A: V \rightarrow V^{*}$ as

$$
A u=\operatorname{div} \mathcal{A} \nabla u \quad(u \in V)
$$

In what follows, $\mathcal{A} \xi$ is an $(m \times n)$-matrix with the components $(\mathcal{A} \xi)_{i j}=a_{i j k l} \xi_{k l},(\mathcal{A} \xi, \eta)$ is a bilinear form such that $(\mathcal{A} \xi, \eta)=a_{i j k l} \xi_{k l} \eta_{i j}\left(\xi, \eta \in \mathbb{R}^{m \times n}\right.$, summation over repeated indices is assumed).

Let $\mathcal{A}^{1}$ and $\mathcal{A}^{2}$ satisfy (5). We will also use the following notations:

$$
\begin{aligned}
\Lambda & =\left\{\chi \in L^{\infty}(\Omega) \mid \chi(x) \in\{0,1\} \text { for a.e. } x \in \Omega\right\} \\
\Gamma & =\left\{\theta \in L^{\infty}(\Omega) \mid 0 \leq \theta(x) \leq 1 \text { for a.e. } x \in \Omega\right\} \\
\mathcal{A}_{\theta} & =\theta \mathcal{A}^{1}+(1-\theta) \mathcal{A}^{2} \quad(\theta \in \Gamma) \\
A_{\theta} & =\operatorname{div} \mathcal{A}_{\theta} \nabla .
\end{aligned}
$$

In [4] the following two theorems are proved.

Theorem 2.1. Let $\mathcal{A}^{1}, \mathcal{A}^{2}$ be as mentioned above. If $m \leq n-1$, then

$$
\operatorname{clos}_{s} Z\left(\left\{A_{\chi}\right\}_{\chi \in \Lambda}, f\right)=Z\left(\left\{A_{\theta}\right\}_{\theta \in \Gamma}, f\right) \quad \text { for all } f \in V^{*} \text {. }
$$

Theorem 2.2. Let $g \in\left[L^{2}(\Omega)\right]^{m \times n}$. Suppose that

$$
\operatorname{rang}\left(\mathcal{A}^{1}(x)-\mathcal{A}^{2}(x)\right) g(x) \leq n-1 \quad \text { for a.e. } x \in \Omega
$$

Then the set $\operatorname{clos}_{s} \cup_{\chi \in \Lambda}\left\{\operatorname{div} \mathcal{A}_{\chi} g\right\}$ is convex in $V^{*}$.

It should be noted that in [4] there are proved more general results than Theorems 2.1 and 2.2 . 
Let $m=n$. In this case $\mathcal{A}$ is a fourth order tensor. It is convenient to write the tensor $\mathcal{A}$ as a block matrix

$$
\mathcal{A}=\left(\begin{array}{ccc}
a_{11} & \ldots & a_{1 n} \\
\vdots & \ddots & \vdots \\
a_{n 1} & \ldots & a_{n n}
\end{array}\right)
$$

where $a_{i j}(1 \leq i, j \leq n)$ are $(n \times n)$-matrices. Let $K$ be the class of all $\left(n^{2} \times n^{2}\right)$-matrices $\mathcal{A}$ which are symmetric in the sense

$$
a_{i j}=a_{j i}^{i} \quad(1 \leq i, j \leq n)
$$

where $a^{t}$ means the transposed matrix of $a$. Denote by $K\left(\Omega, \nu_{1}, \nu_{2}\right)$ the class of all $\left(n^{2} \times n^{2}\right)$-matrices $\mathcal{A}$ which satisfy (5) and belong to $K$.

Our goal is to prove the following

Theorem 2.3. Let $m=n=2$. Assume that $\Omega$ is simply connected. Then there exist $\mathcal{A}^{1}, \mathcal{A}^{2} \in K\left(\Omega, \nu_{1}, \nu_{2}\right)$ such that there does not exist a family $\left\{A_{\alpha}\right\}_{\alpha \in \Xi}$ of operators $A_{\alpha}=\operatorname{div} \mathcal{A}_{\alpha} \nabla \quad\left(\mathcal{A}_{\alpha} \in K\left(\Omega, \nu_{1}, \nu_{2}\right), \alpha \in \Xi\right)$ satisfying

$$
\text { clos, } \bigcup_{\chi \in \Lambda}\left\{A_{\chi} u\right\}=\bigcup_{\alpha \in \equiv}\left\{A_{\alpha} u\right\} \quad \text { for all } u \in V \text {. }
$$

\section{Proof of Theorem 2.3}

The proof is divided into three steps.

Step 1. Let

$$
\mathcal{A}^{1}=\left(\begin{array}{ll}
\left(\begin{array}{ll}
1 & \delta \\
\delta & 1
\end{array}\right) & \left(\begin{array}{ll}
\delta & 0 \\
0 & 0
\end{array}\right) \\
\left(\begin{array}{ll}
\delta & 0 \\
0 & 0
\end{array}\right) & \left(\begin{array}{ll}
1 & 0 \\
0 & 1
\end{array}\right)
\end{array}\right) \quad \text { and } \quad \mathcal{A}^{2}=\left(\begin{array}{ll}
\left(\begin{array}{ll}
1 & 0 \\
0 & 1
\end{array}\right) & \left(\begin{array}{ll}
0 & 0 \\
0 & 0
\end{array}\right) \\
\left(\begin{array}{ll}
0 & 0 \\
0 & 0
\end{array}\right) & \left(\begin{array}{ll}
1 & 0 \\
0 & 1
\end{array}\right)
\end{array}\right)
$$

where $\delta>0$ is such that $\mathcal{A}^{1} \in K\left(\Omega, \nu_{1}, \nu_{2}\right)$ for some $\nu_{1}, \nu_{2}>0$. We will denote by $a_{i j}^{1}$ and $a_{i j}^{2}$ the block elements of $\mathcal{A}^{1}$ and $\mathcal{A}^{2}$, respectively, and we set $a_{i j}^{\theta}=\theta a_{i j}^{1}+(1-\theta) a_{i j}^{2}$.

Let us assume that $\mathcal{B} \in K$ is such that $\operatorname{div} \mathcal{B} \nabla u \in \cup_{\theta \in \Gamma}\left\{A_{\theta} u\right\}$ for all $u \in V$. Then for every $u \in V$ there exists $\theta=\theta(u) \in \Gamma$ such that

$$
\operatorname{div} \mathcal{B} \nabla u=\operatorname{div} \mathcal{A}_{\theta} \nabla u \quad \text { in } V^{*} .
$$

In particular, for every $u \in H_{0}^{1}(\Omega)$ there exists $\theta=\theta(u) \in \Gamma$ for which

$$
\operatorname{div} b_{11} \nabla u=\operatorname{div} a_{11}^{\theta} \nabla u \quad \text { in } H^{-1}(\Omega) .
$$

For $\varphi \in C_{0}^{\infty}(\Omega)$ and $\xi \in \mathbb{R}^{2}$, let us take the sequence

$$
u^{\varepsilon}(x)=\varepsilon \varphi(x) \sin \left(\varepsilon^{-1} x \cdot \xi\right) \quad(\varepsilon>0) .
$$


One can check that

$$
\nabla u^{e}(x)=\varphi(x)\left(\begin{array}{c}
\xi_{1} \cos \left(\varepsilon^{-1} x \cdot \xi\right) \\
\xi_{2} \cos \left(\varepsilon^{-1} x \cdot \xi\right)
\end{array}\right)+\varepsilon \sin \left(\varepsilon^{-1} x \cdot \xi\right) \nabla \varphi(x) .
$$

From (7) we obtain

$$
\int_{\Omega}\left(b_{11} \nabla u^{\varepsilon}, \nabla u^{\varepsilon}\right) d x=\int_{\Omega}\left(a_{11}^{\theta_{\varepsilon}} \nabla u^{\varepsilon}, \nabla u^{\varepsilon}\right) d x
$$

where $\theta_{\varepsilon}=\theta\left(u^{\varepsilon}\right)$. By passing to the limit as $\varepsilon \rightarrow 0$, we obtain

$$
\int_{\Omega} \inf _{\theta \in \Gamma}\left(a_{11}^{\theta}(x) \xi, \xi\right) \varphi^{2} d x \leq \int_{\Omega}\left(b_{11} \xi, \xi\right) \varphi^{2} d x \leq \int_{\Omega} \sup _{\theta \in \Gamma}\left(a_{11}^{\theta}(x) \xi, \xi\right) \varphi^{2} d x
$$

In other words, we have

$$
\inf _{\theta \in \Gamma}\left(a_{11}^{\theta}(x) \xi, \xi\right) \leq\left(b_{11}(x) \xi, \xi\right) \leq \sup _{\theta \in \Gamma}\left(a_{11}^{\theta}(x) \xi, \xi\right)
$$

for a.e. $x \in \Omega$ and all $\xi \in \mathbb{R}^{2}$. By choosing in this inequality $\xi$ as $(1,0),(0,1)$ and $(1,1)$, we deduce that $b_{11}=\left(\begin{array}{cc}1 & \theta_{1} \delta \\ \theta_{1} \delta & 1\end{array}\right)$ for some $\theta_{1} \in \Gamma$. Similarly, we see that $b_{22}=\left(\begin{array}{ll}1 & 0 \\ 0 & 1\end{array}\right)$.

Now, suppose that $b_{12}=\left(\begin{array}{ll}a & b \\ c & d\end{array}\right)$. Using the same reasoning as above, we obtain

$$
0 \leq a(x) \xi_{1}^{2}+(b(x)+c(x)) \xi_{1} \xi_{2}+d(x) \xi_{2}^{2} \leq \delta \xi_{1}^{2}
$$

for a.e. $x \in \Omega$ and all $\xi \in \mathbb{R}^{2}$. Hence $d=0, b=-c$ and $a=\theta_{2} \delta$ for some $\theta_{2} \in \Gamma$. Since $b_{12}=b_{21}^{t}$, we finally have

$$
\mathcal{B}=\left(\begin{array}{cc}
\left(\begin{array}{cc}
1 & \theta_{1} \delta \\
\theta_{1} \delta & 1
\end{array}\right) & \left(\begin{array}{cc}
\theta_{2} \delta & b \\
-b & 0
\end{array}\right) \\
\left(\begin{array}{cc}
\theta_{2} \delta & -b \\
b & 0
\end{array}\right) & \left(\begin{array}{cc}
1 & 0 \\
0 & 1
\end{array}\right)
\end{array}\right)
$$

Let us prove that $\theta_{1}=\theta_{2}$ and $b=$ const. First of all, let us establish that $b=$ const. It is clear that $\operatorname{div} b_{21} \nabla u=\operatorname{div} a_{21}^{\theta(u)} \nabla u$ for all $u \in H_{0}^{1}(\Omega)$. Consequently,

$$
\frac{\partial}{\partial x_{2}}\left(b \frac{\partial u}{\partial x_{1}}\right)-\frac{\partial}{\partial x_{1}}\left(\frac{\partial u}{\partial x_{2}}\right)=\delta \frac{\partial}{\partial x_{1}}\left(\left(\theta(u)-\theta_{2}\right) \frac{\partial u}{\partial x_{1}}\right)
$$

for all $u \in H_{0}^{1}(\Omega)$. We will show that $b(x)=b\left(x_{2}\right)$ for a.e. $x \in \Omega$. To do this, let $\Omega^{\prime} \subset \subset \Omega$ and $u^{0}=\rho_{1} x_{2}$ where $\rho_{1} \in C_{0}^{\infty}(\Omega)$ with $\rho_{1}=1$ on $\Omega^{\prime}$. Using (8), we obtain

$$
\int_{\Omega} b\left(\frac{\partial u}{\partial x_{1}} \frac{\partial \varphi}{\partial x_{2}}-\frac{\partial u}{\partial x_{2}} \frac{\partial \varphi}{\partial x_{1}}\right) d x=\delta \int_{\Omega}\left(\theta(u)-\theta_{2}\right) \frac{\partial u}{\partial x_{1}} \frac{\partial \varphi}{\partial x_{1}} d x
$$


for all $\varphi \in C_{0}^{\infty}(\Omega)$. By replacing $u^{0}$ in this relation, we see that

$$
\int_{\Omega} b \frac{\partial \varphi}{\partial x_{1}} d x=0 \quad \text { for all } \varphi \in C_{0}^{\infty}\left(\Omega^{\prime}\right) .
$$

Since $\Omega^{\prime} \subset \subset \Omega$ is arbitrary, $b(x)=b\left(x_{2}\right)$ for a.e. $x \in \Omega$. From (8) it follows that

$$
\frac{\partial b}{\partial x_{2}} \frac{\partial u}{\partial x_{1}}=\delta \frac{\partial}{\partial x_{1}}\left(\left(\theta(u)-\theta_{2}\right) \frac{\partial u}{\partial x_{1}}\right)
$$

in $\mathcal{D}^{\prime}(\Omega)$, for all $u \in C_{0}^{\infty}(\Omega)$. Since

$$
\frac{\partial}{\partial x_{1}}\left(\frac{\partial b}{\partial x_{2}} u\right)=\frac{\partial b}{\partial x_{2}} \frac{\partial u}{\partial x_{1}},
$$

we have

$$
\frac{\partial b}{\partial x_{2}} u=\delta\left(\theta(u)-\theta_{2}\right) \frac{\partial u}{\partial x_{1}}+f_{u}\left(x_{2}\right)
$$

where $f_{u}$ is a function depending on $u$.

We will show that $\frac{\partial b}{\partial x_{2}}=0$ in $\mathcal{D}^{\prime}(\Omega)$. For this, let us prove that for every point $\left(\hat{x}_{1}, \hat{x}_{2}\right) \in \Omega$ there exists its neighbourhood $U \subset \Omega$ such that $\frac{\partial b}{\partial x_{2}}=0$ in $\mathcal{D}^{\prime}(U)$. Let $\left(\hat{x}_{1}, \hat{x}_{2}\right) \in \Omega$. Without loss of generality, we can assume that $\left(\hat{x}_{1}, \hat{x}_{2}\right)=\left(0, \hat{x}_{2}\right)$. Denote $U=(-\omega, \omega) \times\left(\hat{x}_{2}-\omega, \hat{x}_{2}+\omega\right)$. It is clear that $U \subset \Omega$ for suitable $\omega>0$. Set

$$
u_{C}(x)= \begin{cases}\exp \left(\frac{C}{x_{1}}\right) \rho_{2}(x) & \text { for } x_{1}>0 \\ 0 & \text { for } x_{1} \leq 0\end{cases}
$$

where $\rho_{2} \in C_{0}^{\infty}(\Omega), \rho_{2}=1$ on $U$ and $C<0$. From (9) we have

$$
-\frac{x_{1}^{2}}{C} \frac{\partial b}{\partial x_{2}}+x_{1}^{2} \exp \left(-\frac{C}{x_{1}}\right) \frac{f_{C}\left(x_{2}\right)}{C}=\delta\left(\theta_{C}-\theta_{2}\right)
$$

in $\mathcal{D}^{\prime}\left(\left\{x \in U \mid x_{1}>0\right\}\right)$ where $\theta_{C}=\theta\left(u_{C}\right)$ and $f_{C}=f_{u_{C}}$. Let us fix $C<0$. If $f_{C} \neq 0$ in $\mathcal{D}^{\prime}\left(\hat{x}_{2}-\omega, \hat{x}_{2}+\omega\right)$, then there exists $\varphi \in C_{0}^{\infty}\left(\hat{x}_{2}-\omega, \hat{x}_{2}+\omega\right)$ such that $\left(f_{C}, \varphi\right) \neq 0$ where $(\cdot, \cdot)$ denotes the duality pairing between $\mathcal{D}^{\prime}$ and $\mathcal{D}$. Let $\left\{\varphi^{\beta}\right\}_{0<\beta<\omega}$ be a sequence of functions $\varphi^{\beta}$ such that $\varphi^{\beta}=1$ on $\left(\frac{\beta}{3}, \frac{\beta}{2}\right), 0 \leq \varphi^{\beta} \leq 1$ on $(0, \beta)$ and $\varphi^{\beta} \in C_{0}^{\infty}(0, \beta)$. For $\left(x_{1}, x_{2}\right) \in\left\{x \in U \mid x_{1}>0\right\}$, define $\psi^{\beta}\left(x_{1}, x_{2}\right)=\varphi^{\beta}\left(x_{1}\right) \varphi\left(x_{2}\right)$. From (10) we have

$$
\begin{gathered}
-\left(\frac{\partial b}{\partial x_{2}}, \varphi\right) \int_{0}^{\beta} \frac{x_{1}^{2}}{C} \varphi^{\beta}\left(x_{1}\right) d x_{1}+\frac{1}{C}\left(f_{C}, \varphi\right) \int_{0}^{\beta} x_{1}^{2} \exp \left(-\frac{C}{x_{1}}\right) \varphi^{\beta}\left(x_{1}\right) d x_{1} \\
=\delta \int_{\left\{x \in U \mid x_{1}>0\right\}}\left(\theta_{C}-\theta_{2}\right) \psi^{\beta} d x
\end{gathered}
$$

for all $0<\beta<\omega$. By passing to the limit as $\beta \rightarrow+0$, we see that the first integral on the left-hand side and the integral on the right-hand side tend to 0 , but the second 
integral on the left-hand side tends to $+\infty$. This is a contradiction with $f_{C} \neq 0$ in $\mathcal{D}^{\prime}\left(\hat{x}_{2}-\omega, \hat{x}_{2}+\omega\right)$. Hence $f_{C}=0$ in $\mathcal{D}^{\prime}\left(\hat{x}_{2}-\omega, \hat{x}_{2}+\omega\right)$ for all $C<0$. If $\frac{\partial b}{\partial x_{2}} \neq 0$ in $\mathcal{D}^{\prime}\left(\hat{x}_{2}-\omega, \hat{x}_{2}+\omega\right)$, there exists $\varphi \in C_{0}^{\infty}\left(\hat{x}_{2}-\omega, \hat{x}_{2}+\omega\right)$ such that $\left(\frac{\partial b}{\partial x_{2}}, \varphi\right) \neq 0$. Let $\sigma \in C_{0}^{\infty}(0, \omega)$ be a non-vanishing function. Then, by passing to the limit as $C \rightarrow-0$, we see that

$$
\left(\frac{\partial b}{\partial x_{2}}, \varphi\right) \int_{0}^{\omega} x_{1}^{2} \sigma\left(x_{1}\right) d x_{1}=0 .
$$

This is a contradiction with $\frac{\partial b}{\partial x_{2}} \neq 0$. Thus, $b=$ const on $\Omega$.

Now let us prove that $\theta_{1}=\theta_{2}$. We have

$$
\left(\mathcal{B}-\mathcal{A}_{\theta}\right) \nabla u=\delta\left(\begin{array}{cc}
\left(\theta_{1}-\theta\right) \frac{\partial u_{1}}{\partial x_{2}}+\left(\theta_{2}-\theta\right) \frac{\partial u_{2}}{\partial x_{1}} & \left(\theta_{1}-\theta\right) \frac{\partial u_{1}}{\partial x_{1}} \\
\left(\theta_{2}-\theta\right) \frac{\partial u_{1}}{\partial x_{1}} & 0
\end{array}\right) .
$$

Since for every $v \in V$ there corresponds $\theta=\theta(v)$ such that $\operatorname{div} \mathcal{B} \nabla v=\operatorname{div} \mathcal{A}_{\theta} \nabla v$, we obtain that for every $u \in H_{0}^{1}(\Omega)$ there exists $\theta=\theta(u)$ such that

$$
\int_{\Omega}\left(\theta_{2}-\theta\right) \frac{\partial u}{\partial x_{1}} d x=0 \quad \text { and } \quad \int_{\Omega}\left(\theta_{1}-\theta\right) \frac{\partial u}{\partial x_{1}} d x=0 .
$$

Therefore,

$$
\int_{\Omega}\left(\theta_{1}-\theta_{2}\right) \frac{\partial u}{\partial x_{1}} d x=0 \quad \text { for all } u \in H_{0}^{1}(\Omega) \text {. }
$$

In other words, $\theta_{1}-\theta_{2}=g\left(x_{2}\right)$ on $\Omega$. From (6) it follows that for each $u=\left(u_{1}, u_{2}\right) \in V$ there exists $\theta=\theta(u)$ such that

$$
\left.\begin{array}{rl}
\frac{\partial}{\partial x_{1}}\left(\left(\theta_{1}-\theta\right) \frac{\partial u_{1}}{\partial x_{2}}\right)+\frac{\partial}{\partial x_{1}}\left(\left(\theta_{2}-\theta\right) \frac{\partial u_{2}}{\partial x_{1}}\right)+\frac{\partial}{\partial x_{2}}\left(\left(\theta_{1}-\theta\right) \frac{\partial u_{1}}{\partial x_{1}}\right) & =0 \\
\frac{\partial}{\partial x_{1}}\left(\left(\theta_{2}-\theta\right) \frac{\partial u_{1}}{\partial x_{1}}\right) & =0 .
\end{array}\right\}
$$

Let $p=\theta_{1}-\theta$. Then from (11) we see that ( $p$ depends on a choice of $\varphi$ )

$$
\left.\begin{array}{rl}
\frac{\partial p}{\partial x_{1}} \frac{\partial \varphi}{\partial x_{2}}+\frac{\partial p}{\partial x_{2}} \frac{\partial \varphi}{\partial x_{1}} & =-2 p \frac{\partial^{2} \varphi}{\partial x_{1} \partial x_{2}} \\
\frac{\partial p}{\partial x_{1}} \frac{\partial \varphi}{\partial x_{1}} & =\left(g\left(x_{2}\right)-p\right) \frac{\partial^{2} \varphi}{\partial x_{1}^{2}}
\end{array}\right\} \quad \text { in } \mathcal{D}^{\prime}(\Omega)
$$

for all $\varphi \in C_{0}^{\infty}(\Omega)$.

Let $\Omega^{\prime} \subset \subset \Omega$. Take $\varphi=\left(x_{1}-r\right)^{2} \rho_{3}$, with $\rho_{3} \in C_{0}^{\infty}(\Omega)$ and $\rho_{3}=1$ on $\Omega^{\prime}$, where $r>0$ is such that $\Omega \subset \subset(-r, r) \times(-r, r)$. Hence

$$
\left.\begin{array}{l}
\frac{\partial p}{\partial x_{2}}\left(x_{1}-r\right)=0 \\
\frac{\partial p}{\partial x_{1}}\left(x_{1}-r\right)=g\left(x_{2}\right)-p
\end{array}\right\} \quad \text { in } \mathcal{D}^{\prime}\left(\Omega^{\prime}\right)
$$


Therefore, $p=p\left(x_{1}\right)$ and $g\left(x_{2}\right)=p\left(x_{1}\right)+\left(x_{1}-r\right) \frac{\partial p}{\partial x_{1}}$ in $\Omega^{\prime}$. Thus, $g=$ const $=C_{1}$ on $\Omega$. Let us prove that $C_{1}=0$. To do this, let $\Omega^{\prime} \subset \subset \Omega$. Set $\varphi=\left(x_{1}-r\right)^{2}\left(x_{2}-r\right) \rho_{4}$, where $\rho_{4} \in C_{0}^{\infty}(\Omega)$ and $\rho_{4}=1$ on $\Omega^{\prime}$. From (12) we derive

$$
\left.\begin{array}{rl}
\frac{\partial p}{\partial x_{1}}\left(x_{1}-r\right)^{2}+2 \frac{\partial p}{\partial x_{2}}\left(x_{1}-r\right)\left(x_{2}-r\right) & =-4 p\left(x_{1}-r\right) \\
\frac{\partial p}{\partial x_{1}}\left(x_{1}-r\right)\left(x_{2}-r\right) & =\left(C_{1}-p\right)\left(x_{2}-r\right)
\end{array}\right\} \quad \text { in } \mathcal{D}^{\prime}\left(\Omega^{\prime}\right)
$$

Hence we obtain

$$
\left.\begin{array}{c}
\frac{\partial p}{\partial x_{1}}\left(x_{1}-r\right)=C_{1}-p \\
2 \frac{\partial p}{\partial x_{2}}\left(x_{2}-r\right)=-C_{1}-3 p
\end{array}\right\} \quad \text { in } \mathcal{D}^{\prime}\left(\Omega^{\prime}\right) .
$$

By differentiating these relations, we deduce

$$
\left.\begin{array}{c}
\frac{\partial^{2} p}{\partial x_{2} \partial x_{1}}\left(x_{1}-r\right)=-\frac{\partial p}{\partial x_{2}} \\
2 \frac{\partial^{2} p}{\partial x_{1} \partial x_{2}}\left(x_{2}-r\right)=-3 \frac{\partial p}{\partial x_{1}}
\end{array}\right\} \quad \text { in } \mathcal{D}^{\prime}\left(\Omega^{\prime}\right)
$$

By multiplying these relations by $2\left(x_{2}-r\right)$ and $x_{1}-r$, respectively, and subtracting the results, we see that

$$
2\left(x_{2}-r\right) \frac{\partial p}{\partial x_{2}}=3\left(x_{1}-r\right) \frac{\partial p}{\partial x_{1}} \quad \text { in } \mathcal{D}^{\prime}\left(\Omega^{\prime}\right) .
$$

By (13), we obtain

$$
\left.\begin{array}{c}
3\left(x_{1}-r\right) \frac{\partial p}{\partial x_{1}}=-C_{1}-3 p \\
\frac{\partial p}{\partial x_{1}}\left(x_{1}-r\right)=C_{1}-p
\end{array}\right\} \quad \text { in } \mathcal{D}^{\prime}\left(\Omega^{\prime}\right)
$$

Hence $C_{1}=0$.

Thus, we have proved that if $\operatorname{div} \mathcal{B} \nabla u \in \bigcup_{\theta \in \Gamma}\left\{A_{\theta} u\right\}$ for all $u \in V$ and $\mathcal{B} \in K$, then $\mathcal{B}=\mathcal{A}_{\theta}+c \mathcal{T}$ where $c$ is a constant and

$$
\mathcal{T}=\left(\begin{array}{cc}
\left(\begin{array}{cc}
0 & 0 \\
0 & 0
\end{array}\right) & \left(\begin{array}{cc}
0 & -1 \\
1 & 0
\end{array}\right) \\
\left(\begin{array}{cc}
0 & 1 \\
-1 & 0
\end{array}\right) & \left(\begin{array}{ll}
0 & 0 \\
0 & 0
\end{array}\right)
\end{array}\right)
$$

It is clear that $\operatorname{div} \mathcal{T} \nabla u=0$ for all $u \in V$. 
Step 2. Let $A_{\theta}$ be such that $A_{\theta} u \in \operatorname{clos}_{s} \cup_{\chi \in \Lambda}\left\{A_{\chi} u\right\}$ for all $u \in V$. Let us assume that there exist a measurable set $M \subset \Omega$ with meas $M>0$ and a constant $\varepsilon>0$ such that $\varepsilon \leq \theta(x) \leq 1-\varepsilon$ for a.e. $x \in M$. Straightforward calculations give

$$
\left(\mathcal{A}^{1}-\mathcal{A}^{2}\right) \nabla u=\delta\left(\begin{array}{cc}
\frac{\partial u_{1}}{\partial x_{2}}+\frac{\partial u_{2}}{\partial x_{1}} & \frac{\partial u_{1}}{\partial x_{1}} \\
\frac{\partial u_{1}}{\partial x_{1}} & 0
\end{array}\right)
$$

It is obvious that there is $\varphi \in\left[C_{0}^{\infty}(\Omega)\right]^{2}$ such that there exists an open ball

$$
U \subset\left\{x \in \Omega \mid \operatorname{det}\left(\mathcal{A}^{1}-\mathcal{A}^{2}\right) \nabla \varphi(x)<R<0\right\}
$$

with meas $U \cap M>0$. Since $A_{\theta} \varphi$ belongs to clos, $\cup_{\chi} \in \Lambda\left\{A_{\chi} \varphi\right\}$, there exists a sequence $\left\{\chi_{k}\right\} \subset \Lambda$ for which $A_{\chi_{k}} \varphi \rightarrow A_{\theta} \varphi$ in $V^{*}$. In other words, we have

$$
\left(\mathcal{A}^{1}-\mathcal{A}^{2}\right) \nabla \varphi \operatorname{grad}\left(\chi_{k}-\theta\right)+\left(\chi_{k}-\theta\right) \operatorname{div}\left(\mathcal{A}^{1}-\mathcal{A}^{2}\right) \nabla \varphi \rightarrow 0 \quad \text { in } V^{*} .
$$

Without loss of generality, we can assume that $\chi_{k} \dot{*} \bar{\theta}$ in $L^{\infty}(\Omega)$. Hence $\operatorname{div} \mathcal{A}_{\theta} \nabla \varphi=$ $\operatorname{div} \mathcal{A}_{\bar{\theta}} \nabla \varphi$ in $V^{*}$. Therefore, $\left(\mathcal{A}_{\theta}-\mathcal{A}_{\bar{\theta}}\right) \nabla \varphi=\sigma \nabla v$ (see [8]) for some $v \in\left[H^{1}(\Omega)\right]^{2}$ where $\sigma$ is an operator on the set of all $(2 \times 2)$-matrices defined by

$$
\sigma\left(\begin{array}{ll}
\xi_{11} & \xi_{12} \\
\xi_{21} & \xi_{22}
\end{array}\right)=\left(\begin{array}{ll}
-\xi_{12} & \xi_{11} \\
-\xi_{22} & \xi_{21}
\end{array}\right)
$$

Since $\varphi \in\left[C_{0}^{\infty}(\Omega)\right]^{2}$, we can suppose that $v$ belongs to $V$. Hence

$$
\int_{\Omega} \operatorname{det}\left(\mathcal{A}_{\theta}-\mathcal{A}_{\bar{\theta}}\right) \nabla \varphi d x=0
$$

This yields

$$
\int_{\Omega}(\theta-\bar{\theta})^{2} \operatorname{det}\left(\mathcal{A}^{1}-\mathcal{A}^{2}\right) \nabla \varphi d x=0 .
$$

By the assumption on the sign of the determinant, we obtain that $\theta(x)=\bar{\theta}(x)$ for a.e. $x \in U$. From this, (14) and compactness of the embedding $L^{2}(U) \hookrightarrow H^{-1}(U)$ it follows that

$$
\left(\mathcal{A}^{1}-\mathcal{A}^{2}\right) \nabla \varphi \operatorname{grad}\left(\chi_{k}-\theta\right) \rightarrow 0 \quad \text { in }\left[H^{-1}(U)\right]^{2} .
$$

In other words, $\operatorname{grad}\left(\chi_{k}-\theta\right) \rightarrow 0$ in $\left[H^{-1}(U)\right]^{2}$. Using Nečas's inequality (see [1]; cf. also [7]), we have

$$
\left\|\chi_{k}-\theta-M_{k}\right\|_{L^{2}(U)} \leq C_{2}\left\|\operatorname{grad}\left(\chi_{k}-\theta\right)\right\|_{\left[H^{-1}(U)\right]^{2}}
$$

where $M_{k}=(\text { meas } U)^{-1} \int_{U}\left(\chi_{k}-\theta\right) d x$. Hence we obtain that $\theta$ is a characteristic function on $U$. This is a contradiction with the assumption on $\theta$. Thus, if $\theta$ is not a characteristic function, the inclusion $A_{\theta} u \in \operatorname{clos}, \cup_{\chi \in \Lambda}\left\{A_{\chi} u\right\}$ can not be satisfied for all $u \in V$. 
Step 3. Now we will prove that there exists $u \in V$ for which the set $\cup_{\chi} \in \Lambda\left\{A_{\chi} u\right\}$ is not closed in $V^{*}$. Let $u_{1}=x_{2} \rho$ and $u_{2}=0$, with $\rho \in C_{0}^{\infty}(\Omega)$ and $\rho=1$ on $\Omega^{\prime} \subset \subset \Omega$. Then, for $u=\left(u_{1}, u_{2}\right)$,

$$
\operatorname{rang}\left(\mathcal{A}^{1}-\mathcal{A}^{2}\right) \nabla u=\operatorname{rang}\left(\begin{array}{cc}
\frac{\partial u_{1}}{\partial x_{2}}+\frac{\partial u_{2}}{\partial x_{1}} & \frac{\partial u_{1}}{\partial x_{1}} \\
\frac{\partial u_{1}}{\partial x_{1}} & 0
\end{array}\right)=1 \quad \text { for all } x \in \Omega^{\prime}
$$

Assume that the set $U_{\chi \in \Lambda}\left\{A_{\chi} u\right\}$ is closed in $V^{*}$. Then, by Theorem 2.2 , this set contains the set

$$
\left\{A_{\theta} u \mid \theta \in \Gamma \text { and } \theta(x)=0 \text { or } \theta(x)=1 \text { in } \Omega \backslash \Omega^{\prime}\right\} \text {. }
$$

It means that for every $\theta \in \Gamma$ there exists $\chi=\chi(\theta) \in \Lambda$ satisfying the equality

$$
\frac{\partial}{\partial x_{1}}\left((\chi-\theta) \frac{\partial u_{1}}{\partial x_{2}}\right)+\frac{\partial}{\partial x_{1}}\left((\chi-\theta) \frac{\partial u_{2}}{\partial x_{1}}\right)+\frac{\partial}{\partial x_{2}}\left((\chi-\theta) \frac{\partial u_{1}}{\partial x_{1}}\right)=0
$$

in $H^{-1}\left(\Omega^{\prime}\right)$. Taking into account the form of $u_{1}$ and $u_{2}$, we obtain that $\frac{\partial}{\partial x_{1}}(\chi-\theta)=0$ in $H^{-1}\left(\Omega^{\prime}\right)$. This gives that $\chi-\theta=f_{\theta}\left(x_{2}\right)$ for a.e. $x \in \Omega^{\prime}$.

Let $\theta=\left|\sin x_{1}\right|$ where $|\cdot|$ denotes the absolute value. Let $M$ be the intersection of Lebesgue sets of $\chi$ and $f_{\theta}$. If we consider the cuts of $M$ by the sets $\Upsilon_{c}=\left\{x \mid x_{2}=c\right\}$ (c $\in$ $\mathbb{R}$ ), it is evident that there is $c_{0}$ for which the set $\Pi=M \cap \Upsilon_{c_{0}}$ has a positive Lebesgue measure on the real line. For points belonging to $\Pi$, we have $\chi\left(x_{1}, c_{0}\right)=\left|\sin x_{1}\right|+f_{\theta}\left(c_{0}\right)$. In other words, $\left|\sin x_{1}\right|-\left|\sin x_{1}^{\prime}\right| \in\{-1,1,0\}$ for all $\left(x_{1}, c_{0}\right),\left(x_{1}^{\prime}, c_{0}\right) \in \Pi$. But this is impossible since the function $\left|\sin x_{1}\right|-\left|\sin x_{1}^{\prime}\right|$ takes other values, too, when $\left(x_{1}, c_{0}\right)$ and $\left(x_{1}^{\prime}, c_{0}\right)$ vary in $\Pi$. Thus, we have shown that the set $\cup_{\chi \in \Lambda}\left\{A_{\chi} u\right\}$ can not be closed in $V^{*}$.

Combining the just proved three steps, we obtain the theorem

Acknowledgement. The author would like to thank Professor Uldis Raitums for introducing to the problem and for his encouragement and valuable mathematical discussions.

\section{References}

[1] Kohn, R. V. and G. Strang: Optimal design and relaxation of variational problems, Parts I - III. Comm. Pure Appl. Math. 39 (1986), 113 - 137, 139 - 182 and 353 - 377.

[2] Nečas, J.: Les méthodes directes en théorie des équations elliptiques. Prague: Academia 1967.

[3] Raitums, U.: Optimal Control Problems for Elliptic Equations (in Russian). Riga: Zinatne 1989.

[4] Raitums, U.: On the projections of multivalued maps. J. Optim. Theory Appl. 92 (1997), $633-660$.

[5] Raitums, U.: Lecture Notes on G-convergence, Convexification and Optimal Control Problems for Elliptic Equations (Lect. Notes: Vol. 39). Jyväskylä: University, Dept. Math. 1997. 
[6] Tartar, L.: Remarks on optimal design problems. In: Calculus of Variations, Homogenization and Continuum Mechanics (eds.: G. Buttazzo, G. Bouchitte and P. Suquet). Singapore: World Sci. 1994, pp. $279-296$.

[7] Temam, R.: Navier-Stokes Equations. Amsterdam: North-Holland Publ. Comp. 1979.

[8] Zhikov, V. V., Kozlov, S. M. and O. A. Olejnik: Homogenization of Differential Operators and Integral Functionals. Berlin: Springer-Verlag 1994.

Received 15.05.1997; in revised form 03.06.1998 